\title{
L'étude des champs associatifs du français : création d'un dictionnaire des normes associatives
}

\author{
Michèle Debrenne \\ Université d'Etat Novossibirsk \\ Claude Frey \& Mary-Annick Morel \\ Université Paris 3, EA 1483
}

\section{Introduction}

L'intérêt croissant pour l'ethnopsycholinguistique et la communication interculturelle est conditionné par la quantité croissante de contacts interethniques. La description, l'analyse et la prise en compte de «l'image du monde » de tel ou tel peuple et sa confrontation avec la vision du monde des autres permet d'expliquer pourquoi les différentes formes de contact linguistique telles que l'apprentissage d'une langue étrangère, la traduction ou la communication interpersonnelle directe ne s'opèrent pas avec la meilleure efficacité, et de donner des recommandations quant à leur optimisation.

Ainsi qu'il est avéré dans les modèles WAN (Word Association Norms) et WAT (Word Association Thesaurus) il existe un lien entre les associations verbales, représentatives de la conscience linguistique, et les perceptions ethnologiques et socioculturelles du monde. L'école de psycholinguistique russe (voir notamment les travaux de Yu. Karaulov [Караулов 1999], Е. F. Tarasov [Тарасов 2002, 2004], N. V. Ufimsteva [Уфимцева 2000, 2002]), propose un cadre méthodologique commode pour l'étude de la conscience linguistique pouvant être appliqué à d'autres langues, ce qui a effectivement été réalisé : ces dernières années ont été publiés les dictionnaires des normes associatives de l'espagnol [Puig, Karaulov, Cherkasova 2001], de l'allemand [Уфимцева 2004], du polonais, des langues slaves [Уфимцева et al. 2004]. A l'heure actuelle il n'existe malheureusement aucun ouvrage ou recherche de la sorte concernant le français. L'étude entreprise par notre équipe internationale franco-russe se propose de pallier cette absence.

\section{Historique de la création de dictionnaires associatifs}

Les premiers à utiliser l'expérience associative dans l'étude du discours ont été les chercheurs allemands A. Thumb et K. Marbe [1901]. A l'origine, l'expérience était surtout utilisée pour étudier les processus psychiques, notamment ceux de généralisation ou de reproduction. Dans une seconde étape, elle a été utilisée en linguistique, surtout pour étudier la sémantique, car la question se posait de savoir ce qui dans les expériences était dû aux facteurs psychiques et ce qui était lié à la langue.

Le premier dictionnaire d'associations verbales a été publié en 1910 [Kent, Rosanoff 1910]. Leur liste de stimuli comprenait 100 mots hautement fréquents et ils ont interrogé 1000 personnes. Par la suite, d'autres études ont été entreprises aux Etats-Unis avec la même liste de stimuli, par exemple W. A. Russel et J. J. Jenkins [1954]. Un ouvrage collectif dirigé par L. Postman réunit tous les travaux connus à l'époque sur les normes associatives de l'anglais, du français et de l'allemand [1970]. Les premiers travaux russes d'études des normes associatives se sont concrétisées par un premier dictionnaire des normes associatives du russe en 1977 [Леоньтев 1977].

Depuis les années 90 l'école de psycholinguistique de Moscou a commencé à élaborer une nouvelle base théorique pour les études d'ethnopsycholinguistique, centrées sur l'analyse de la particularité de la conscience linguistique spécifique d'une culture nationale donnée et sur l'idée que l'incompréhension dans la communication interculturelle est due à la différence des consciences 
nationales des acteurs de la communication. La recherche de nouvelles voies de travail a amené les spécialistes à la conception de l'ontologie interculturelle de l'analyse des consciences nationales (ethniques), quand les images de la conscience d'une culture nationale sont analysées en contraste avec celles d'une autre culture. Il a fallu pour cela mettre au point une méthodologie adéquate pour ce type d'analyse contrastive, dans laquelle la langue et la culture sont considérées comme des formes d'existence de la conscience sociale, où se retrouvent l'image de soi (celle de son propre peuple) et l'image de l'autre.

La linguistique contemporaine connait des procédures d'analyse scientifique de la conscience linguistique matérialisée dans les unités de la langues (les mots). Cet ensemble de notions permet de décrire d'une manière très détaillée et différenciée les significations grammaticales, lexicales, stylistiques, occasionnelles, archaïques des lexèmes. Cependant les linguistes ont toujours senti le besoin d'une description plus globale du contenu du mot. Cette étude du contenu global associé à un mot a amené ces dernières années à la création de dictionnaires associatifs, qui contiennent les champs associatifs formés des mots cités par les locuteurs profanes de la langue (non spécialistes, non linguistes) et décrivent les connaissances qu'une société donnée attache à un certain mot à une période donnée de son histoire. Ces champs associatifs ont des caractéristiques uniques :

1. Ils sont opposés aux articles des dictionnaires ordinaires, dans lesquels les définitions sont élaborées par une ou plusieurs personnes sur la base de leurs connaissances personnelles établies au cours de l'analyse d'un certain nombre de contextes.

2. Ils contiennent les connaissances conscientes, des connaissances semi-conscientes et des connaissances inconscientes.

3. Ils contiennent une partie générale, constante, dans laquelle sont réunies les connaissances partagées par tous, et une partie représentée par les connaissances individuelles, obtenues par des réponses non répétives, et qui est changeante dans le temps. Ainsi, en français, le stimulus pain provoquera un grand nombre de réactions associatives vin, car cette association fait partie des connaissances partagées par tous, alors qu'une personne souffrant de maladie coeliaque pourra réagir par maladie ou allergie, ce qui sera pris en compte dans les réactions individuelles. De même, la réaction islamique au stimulus foulard est explicable par l'actualité : une expérience répétée à une autre période n'obtiendra peut-être pas le même nombre de réactions de ce genre.

La linguistique considère que la signification d'un mot est une valeur concrète, finie, transparente, qui peut être décrite comme une constante. Elle part de la présomption que la signification d'un mot est une connaissance consciente que le créateur d'un dictionnaire peut décrire de manière précise. Autrement dit les définitions des dictionnaires excluent la partie inconsciente des connaissances sur les mots, se basant notamment sur le fait que l'accès en est difficile.

Les dictionnaires ordinaires permettent à leurs utilisateurs d'obtenir des connaissances formées d'après des règles théoriques. Cependant les connaissances théoriques, étant le fruit d'une procédure analytique, linéaire et logique, ne représentent pas de manière adéquate le modèle des connaissances de l'utilisateur profane, dont la connaissance se crée au cours de processus non linéaires, parallèles et qui se déroulent simultanément au niveau conscient et inconscient. Les modèles réticulaires de conservation er de recherche des mots dans la mémoire verbale, actuellement les plus adéquats, montrent que la recherche du mot nécessaire lors de la production et la compréhension du discours s'accompagnent de l'activation des liens les reliant à d'autres mots. La compréhension du mot ou de l'énoncé se déroule en grande partie d'une manière inconsciente et entraine la formation d'une connaissance construite différement de la connaissance théorique, laquelle est réversible et déterminée.

L'analyse de la perception du langage montre qu'un seul et même énoncé est perçu différemment par différents destinataires, car lors de la compréhension ils utilisent non seulement leurs connaissances conscientes, mais aussi des connaissances inconscientes. 
L'élaboration de champs associatifs et la création de dictionnaires associatifs sont une tentative de montrer et de délimiter les connaissances (conscientes et inconscientes) utilisées par les locuteurs d'une langue et d'une culture données (dans notre cas, le français) lors de la compréhension d'un mot.

\section{Bases psycholinguistiques de l'étude des associations verbales}

La conscience linguistique ne peut être analysée que dans ses résultats, les objets ou quasi-objets culturels. La recherche de la spécificité nationale et culturelle de la conscience linguistique dépend du statut même de la conscience, moyen de connaissance une culture particulière dans sa forme matérielle, mentale ou dans ses activités. Les études de la conscience linguistique reproduisent ce qui se passe dans la communication interculturelle entre des porteurs de cultures différentes, avec les inévitables problèmes de communication qu'elle comporte (dûs notamment à une compréhension mutuelle incomplète dans des conditions de consciences linguistiques différentes [Тарасов 1998]).

L'étude entreprise de la conscience linguistique des Français est basée sur les principes méthodologiques développés par l'école de psycholinguistique de Moscou, qui développe les travaux de L. S. Vygotsky [1934] et A. A. Leontiev [1965, 1967, 1997]. et s'appuie sur les principes psychologiques suivants: les événements de la réalité sont perçus par l'homme au cours de l'activité de ce dernier et de la communication. Ces événements se fixent dans sa conscience avec les liens de cause à effet, les relations spatio-temporelles et les émotions qu'ont suscitées leur perception comme un ensemble d'images mentales extériorisées sous forme de moyens linguistiques (mots, locutions, expressions, phrases, textes et champs associatifs). La possibilité de créer un dictionnaire associatif est ainsi basée sur la notion psychologique des liens entre les unités de conscience. Celles-ci peuvent être représentées par des images perceptives, des représentations, des notions, des émotions, des sentiments. Chaque culture repose sur un système de significations, de stéréotypes sociaux, de schémas cognitifs. L' « image du monde » peut être commune soit à toute cette société, soit à un de ses groupes socio-culturels. Derrière un mot de la langue maternelle, acquis par l'enfant lors durant le processus d'apprentissage de la langue, il y a une image mentale complexe formée de deux couches : la couche usuelle, où peut être fixée l'image biodynamique de l'action et celle des organes des sens liée à ce mot (le souvenir des actions et des sensations liées à l'idée de "pomme », par exemple) et la couche réfléxive, qui contient la signification et le sens de ce mot. Le «corps » du signe (le mot dans sa forme graphique ou phonétique) est lié à une unité vive de l'image du monde pour chaque culture. Le caractère systématique de la signification reflète le caractère systématique de la culture.

L'ethnopsycholinguistique est une branche de la psycholinguistique qui étudie les variantes des cultures nationales dans leurs opérations langagières, leurs actions linguistiques et leurs actes de discours, dans leur conscience linguistique, c'est-à-dire dans l'utilisation cognitive de la langue et des systèmes sémiotiques équivalents, ainsi que dans l'organisation interne et externe des processus de la communication [Леонтьев, 1999: 192].

L'image du monde est la réflexion du monde matériel par l'intermédiaire des significations concrètes et des schémas cognitifs. Cette image du monde peut être elle-même objet de conscience. Le monde est représenté à l'homme dans un système de significations concrètes, qu'il applique à sa perception du monde. La vision et la perception du monde de chaque peuple est basée sur un système de significations, de stéréotypes et de schémas cognitifs : la conscience de chacun est conditionnée ethniquement : on ne peut pas «reencoder » mécaniquement la vision du monde d'un peuple dans la langue-culture d'un autre.

La conscience linguistique est vue comme l'ensemble des structures de la conscience formée par les connaissances sociales liées aux signes linguistiques ou bien comme des images de la conscience matérialisées par des moyens linguistiques sous forme de lexèmes, syntagmes, idiomes, textes, 
champs associatifs et thésausus associatifs (qui représentent l'ensemble de ces champs). Elle peut être considérée comme le moyen d'étudier une culture étrangère dans sa forme matérielle, mentale et active et comme moyen d'appréhension de sa propre culture. Les images de la conscience linguistique comprennent les connaissances mentales, formées par le sujet lui-même au cours de la communication, les connaissances sensitives, apparues dans la conscience suite au retraitement par l'individu des données perceptives recues des organes des sens au cours de son activité. Les caractères systémiques des objets de la culture ne sont pas observables directement, ils sont symboliques, sémiotiques, donc visibles seulement par celui qui connaît le système dans lequel l'objet culturel donné aquiert ces qualités [Тарасов 1998, 33]. Les objets culturels sont naturels (ne dépendant pas d'une culture particulière), fonctionnels et systémiques (ou intégraux), dépendant d'une culture particulière. Pour une intercompréhension réussie il est indispensable que les acteurs de la communication possèdent des connaissances communes sur la langue utilisée, des savoir-faire communs de communication et des connaissances communes sur le monde sous forme d'images mentales.

\section{Nature de l'expérience}

L'inconscient collectif d'une société peut être étudié au moyen de questionnaires sociologiques. Cependant, dans ce cas, on fixe la métaconscience, c'est-à-dire le jugement que portent les sujets de l'expérience sur leur conscience ethnique. Les expériences ont permis d'analyser les stéréotypes sociaux et culturels peuvent être analysés à l'aide d'expériences malgré qu'ils soient conservés dans l'inconscient collectif et généralisent l'expérience sociale commune aux membres d'une société. L'un des moyens de mise en évidence de la conscience linguistique est l'expérience associative, où les champs associatifs composés des réactions des locuteurs permettent de décrire les caractéristiques des images de la conscience.

Pour construire un dictionnaire associatif il est important que les associations obtenues lors de l'expérience soient formulées par un mot. Un dictionnaire d'associations s'avère donc être un modèle de la conscience et contient des règles d'opération sur les connaissances verbales et non verbales. Le Réseau Associatif Verbal (RAV) reflète les extériorisations verbales de la conscience linguistique proprement dite et non pas une métaconscience, ce qui permet au chercheur d'analyser la conscience ethnique de manière plus précise. Le RAV permet de prendre en compte non seulement les connaissances conscientes sur le monde, mais aussi les connaissances inconscientes. Il est important de tenir compte du fait que le RAV tel qu'il est fixé dans un dictionnaire associatif reflète la conscience linguistique de l'ensemble de la collectivité linguistique étudiée, et non pas celle d'un individu.

La méthode employée pour l'élaboration du dictionnaire associatif du français se base sur la technique répandue en psychologie et psycholinguistique d'expérience associative libre avec fixation de la première réponse. Cette métholologie permet de juger des particularités de la conscience linguistique et des moyens de construction de l'énoncé qui restent généralement inconscients du locuteur et que les autres méthodes ne permettent pas de mettre en évidence. Grâce à l'expérience associative libre il devient possible d'obtenir une information sur les équivalents psychologiques des «champs sémantiques» et de mettre au jour les liaisons sémantiques qui existent réellement dans l'esprit des locuteurs.

Sur le plan qualitatif, la méthode fait appel à toutes les classes verbales pleines (substantifs, verbes, adjectifs, adverbes) dont les associations syntagmatiques ou paradigmatiques révèlent l'interrelation entre les mots, qui apparaissent de façon régulière et au terme de plusieurs centaines de réponses, en nombre fini. Cela permet de cerner par les associations linguistiques les représentations du monde dans une culture donnée.

Dans une première étape il convient d'établir une liste de 1000 mots-stimuli d'après l'analyse de la fréquence des substantifs, adjectifs, adverbes et verbes du français ainsi qu'une liste de critères selon lesquels seront classées les données concernant les participants à l'expérience. Ces listes 
serviront de base à l'établissement des formulaires utilisés lors de l'expérience. On peut par exemple, outre l'âge et la ville, tenir compte des connaissances en langue étrangère ou du bilinguisme éventuel. Chaque mot-stimulus de la liste de départ est présenté 500 fois à des sujets dans différentes régions de France. Les sujets sont des étudiants de différentes spécialités, effectuant leurs études dans des établissements d'enseignement supérieur dans différentes régions du pays. Le choix d'étudiants en tant que sujets d'expérience se justifie par le fait que la plupart des expériences associatives dans le monde ont été effectuées sur ce groupe socio-culturel et selon le même protocole, ce qui permet de comparer les résultats obtenus d'un pays à l'autre. Le français doit être la langue maternelle des personnes interrogées. Il est toutefois possible dans le cadre d'unj étude complémentaire d'interroger des personnes vivant dans le pays mais dont la langue maternelle n'est pas le français pour comparer leur conscience linguistique et les différences éventuelles de leur langue-culture avec celle des locuteurs natifs du français. L'expérience peut se dérouler sur papier (avec entrée ultérieure des données sur ordinateur par un opérateur) ou directement sur ordinateur grâce à un programme simple d'entrée de données.

Ensuite les chercheurs établissent les champs associatifs résultants des 500 réponses données pour chaque stimulus. Ce champ associatif étant composé de réactions fréquentes et de réactions individuelles, on peut notamment, en comparant les réactions fréquentes dans différentes régions du pays, conclure à l'existence d'une spécificité régionale. Le fait que les items soient présentés à un nombre élevé de sujets impliqués dans l'enquête assure la fiabilité des résultats.

\section{Le dictionnaire associatif}

Les résultats des expériences sont présentés sous forme de deux dictionnaires, un dictionnaire direct et un dictionnaire inverse : Dans le dictionnaire direct (du stimulus à la réaction) sont présentées toutes les réactions à un stimulus donné, par ordre de fréquence. Le nombre de réactions identiques est indiqué après chaque mot (ou chaque groupe de mot, s'il y en a plusieurs ; ils sont alors disposés dans l'ordre alphabétique). A la fin de l'article on trouve une série de chiffres, ordinairement séparés par un signe + . Le premier chiffre indique le nombre total de réactions obtenues à ce mot, le second, le nombre de réactions différentes, le troisième est celui des refus et le dernier prend en compte les réactions uniques. Par exemple, dans le dictionnaire des normes associatives de l'espagnol [2001] on peut voir au début de l'article HOMBRE (homme) : HOMBRE : mujer 174; persona 20; ser 16; fuerza, padre, ser humano, sexo 9... L'article se termine par les chiffres suivants: $(573+203+24+144)$, ce qui doit donc être interprété comme suit : sur 573 présentations du stimulus hombre, 24 personnes ont refusé de donner une réponse quelconque. Ce mot a provoqué 203 réactions différentes, dont 144 uniques.

Le dictionnaire inverse (de la réaction au stimulus) est structuré différemment, puisque l'entrée y est représentée par la réaction des sujets de l'expérience aux stimuli. Les chiffres indiquent la fréquence de cette réaction. Ainsi, dans le même dictionnaire, HOMBRE - mujer 88 ; marido 29 ; persona 23... signifie que dans le dictionnaire direct, à l'article mujer le substantif HOMBRE a été donné 88 fois, tandis qu'il a été donné 29 fois comme réaction au stimulus marido et 23 fois à persona. A la fin de l'article on trouve les chiffres suivants : le nombre total de fois où ce mot (ou ce groupe de mot, les réactions pouvant être présentées sous forme de syntagmes) est apparu en tant que réaction, le nombre de stimuli différents qui l'ont provoquée (ce qui correspond au nombre d'articles du dictionnaire direct dans lesquels on peut trouver cette réaction), soit, pour le substantif HOMBRE, 244 et 49 . On note également le nombre de réponses nulles (absence de réaction au stimulus donné).

Cette forme de représentation des données expérimentales dans le dictionnaire associatif permet non seulement d'étudier les différences et ressemblances entre les images mentales des porteurs de cette langue-culture, mais également de mettre en évidence leur vision du monde dans sa complexité. Grâce à divers logiciels on peut déterminer les liens les plus évidents entre les mots, évaluer la force de ces liens et la proximité de sens de mots qu'on peut considérer comme d'autant plus proches qu'ils évoquent les mêmes associations avec la même intensité. 
On considère que les mots qui ont le plus grand nombre de liens associatifs avec d'autres ont une importance particulière pour le sujet d'expérience et pour le peuple locuteur de cette langue en général. Ce sont les notions les plus riches, ces liaisons sont reproduites avec une grande probabilité. Les lexèmes dont les liens associatifs sont les plus forts ne représentent que $2 \% \mathrm{du}$ volume total du lexique d'une langue. Les mots évoqués en tant que réaction au plus grand nombre de stimuli forment le noyau de la conscience linguistique. Ce noyau est différent selon les peuples ; par exemple, pour l'anglais, les 8 premiers mots qui le composent sont : me, man, good, sex, no, money, yes, nothing, tandis que pour le russe, nous obtenons человек ('tchekovek', homme), дом ('dom', maison), нет ('niet', non), хорошо ('khorocho', bien), жизнь ('jizn', vie); плохо ('plokho', mal), большой ('bolchoï', grand), друг ('droug', ami), où l'on voit quelques similitudes, mais aussi de grandes différences.

De plus, l'analyse des relations associatives des mots qui entrent dans ce noyau de la conscience linguistique permet de mettre en évidence des différences encore plus flagrantes entre les langues: ainsi, en russe, le substantif $\partial р y z$ ('droug', ami) occupe, selon les étapes de l'expérience, de la huitième à la dixième place du noyau, il est donc parmi les mots les plus souvent cités. Il a été utilisé comme réaction à 565 lexèmes différents, dont les suivants, classés par groupes thématiques :

верный ('vernyi', fidèle), надежный ('nadiojnyi', sûr), настоящий ('nastojachij', véritable), старый ('staryj', vieux) ;

близкий ('blizkij', proche), любимый ('lioubimyi', préféré), сердечный ('serdetchnyj’, de coeur) ;

лучший ( 'loutchij', meilleur), милый ('milyij', tendre), единственный ('edinstvennyi', unique) и родом из детства ('rodom iz detstva', d'enfance);

товарищ ('tovarichtch, camarade), брат ('brat', frère), собака ('sobaka', chien) ;

Les lexèmes qui ont provoqué le plus souvent le mot ami en tant que réaction sont les équivalents russes de véritable (215 fois), meilleur (156), fidèle (64) et sûr (52).

En attendant d'avoir les chiffres concernant le français, on peut comparer avec l'anglais, où le mot friend occupe la $73^{\text {ème }}$ place. Le plus souvent, il a été cité comme réaction à acquaintance (68)+ aquaintance (67), companion (67), college (50), buddy (36), neighbor (36)+ neigbour (33), ally(35). Ces quelques chiffres permettent de préciser l'impression diffuse des traducteurs, pour lesquels il n'y a jamais de véritable équivalent d'une langue à l'autre. En effet, un texte dans lequel figure le lexème $\partial p y z$, et a fortiori dans celui où il joue un rôle central et peut être considéré comme un motclé, produira en traduction une toute autre impression, évoquera chez le lecteur du texte traduit en anglais des associations tout à fait différentes que celle de l'original. On peut supposer que le champ associatif du substantif français $a m i$ sera à la fois différent de celui de ses équivalents en russe et en anglais. Ainsi, le mot russe ледоход ('ledokhod') évoque pour un locuteur de cette langue une atmosphère de fête, de libération, c'est un évènement attendu, évocateur d'émotions fortes, ce que ne peut pas rendre l'équivalent français débâcle qui évoquera des associations forcément négatives. On est en droit de supposer que les associations données par les Canadiens francophones seront, là aussi, très différentes de celle des Français de France.

On peut également analyser la composition sémantique du noyau de la conscience linguistique et la comparer avec celle d'une autre langue : le noyau de la conscience sémantique de la langue russe comporte des lexèmes représentant les groupes suivants (donnés en traduction ) 


\begin{tabular}{|l|l|}
\hline \multicolumn{1}{|c|}{ Russe } & \multicolumn{1}{c|}{ Anglais } \\
\hline L'homme (10875 réactions) & L'homme (6876) \\
Le travail (les études, les affaires, 5149) & Le corps (3900) \\
La maison (4911) & La nourriture (3418) \\
Le corps humain (3815) & La couleur (3052) \\
La maladie (3311) & La maladie (2601) \\
La couleur (2750) & La maison (2221) \\
La nourriture (2619) & Les animaux (2065) \\
Les animaux (2424) & La guerre (1445) \\
La guerre (1321) & Les vêtements (1272) \\
Les vêtements (1049) & \\
\hline
\end{tabular}

Tableau $\mathrm{N}^{\circ}$ 1. Comparaison des noyaux de conscience linguistique du russe et de l'anglais

On constate que la thématique "travail" est absente du noyau anglais et que malgré des similitudes les préférences sont différentes selon les langues.

Ces comparaisons peuvent concerner plusieurs peuples. Ainsi, dans le tableau ci-dessous on peut observer les correspondances quantitatives entre la composition du noyau de conscience linguistique des Bielorusses, des Bulgares, des Russes et des Ukrainiens :

\begin{tabular}{|l|c|l|l|}
\hline & Bulgares & Russes & Ukrainiens \\
\hline Bielorusses & $53,3 \%$ & $50 \%$ & $63,3 \%-66,7 \%$ \\
\hline Bulgares & & $63,3 \%$ & $60 \%$ \\
\hline Russes & & & $56,6 \%$ \\
\hline
\end{tabular}

Tableau $N^{\circ}$ 2. Comparaison du noyau de conscience linguistiques de quelques peuples slaves

Il sera très intéressant de comparer les résultats obtenus pour le français avec les autres languescultures étudiées selon la même méthodologie, notamment pour des langues pratiquées par des locuteurs vivant dans des civilisations dont les valeurs sont différentes de la civilisation occidentale.

Les dictionnaires les plus intéressants et informatifs sont les thésaurus. On en connaît deux [Kiss 1972] et Русский ассоциативный словарь [1994-1998]. Ce dernier a été créé grâce aux expériences d'association libre effectuées entre 1986 et 1996 sur le territoire de toute la Russie. Les réactions obtenues lors de la première itération d'enquêtes (1986-1991), ont été utilisées en tant que stimuli pour la deuxième itération d'enquêtes (1992-1994), puis les nouvelles réactions ont été utilisées lors de la troisième et dernière itération (1994-1996). On constate qu'au bout de trois itérations il n'apparaît presque plus de nouvelles réactions. Le thésaurus associatif, présenté comme un réseau associatif à plusieurs dimensions, donne une impression précise de la structure et du fonctionnement de la conscience linguistique d'un locuteur moyen de telle ou telle langue, et donc de son "image du monde ». Comme on peut le constater, la création d'un thésaurus associatif s'étale sur de nombreuses années et on peut espérer qu'après la création du dictionnaire associatif du français l'équipe de recherche aura les moyens de poursuivre l'expérience pour obtenir un véritable thésaurus. L'utilisation d'outils informatiques ainsi que la mise en ligne des résultats permettra de faciliter cette tâche et de créer un instrument de travail innovant et fondamental en lexicographie du français.

\section{$6 \quad$ Utilisation des dictionnaires associatifs}

Le premier intérêt de l'étude des réactions associatives est de rendre possible la comparaision avec la structure sémantique du lexique du français (ou plutôt du lexique des locuteurs de cette langue) telle qu'elle est reflétée dans les dictionnaires usuels. L'expérience associative libre permet d'obtenir une information sur les équivalents psychologiques des « champs sémantiques » définis 
par la linguistique traditionnelle et de mettre à nu les liens sémantiques exsistant réellement dans l'esprit des locuteurs.

Le second avantage de l'étude des réactions associatives en français réside dans le fait qu'elles sont conditionnées par les relations syntagmatiques des lexèmes, ce qui a très peu été étudié par les linguistes. Cependant le caractère syntagmatique d'un grand nombre de réactions présente un aspect très prometteur dans l'optique de l'enseignement du français langue étrangère.

Le troisième avantage consiste en ce que les réactions associatives permettent de juger des règles d'occurence conjointe des mots dans le discours (quand un mot en appelle un autre, par exemple exception $\rightarrow$ culturelle, commerce $\rightarrow$ équitable ou téléphone $\rightarrow$ portable pour ne citer que quelques syntagmes récents). En effet, les règles de répartition des mots dans l'expérience associative libre et la probabilité de l'apparition des mêmes mots dans le discours sont très voisines. Comme ces règles d'associations de mots jouent un grand rôle parmi les facteurs conditionnant la génération d'un certain énoncé, l'étude des réactions associatives peut servir de base, en linguistique cognitive, d'une théorie de l'activité langagière et de l'acquisition du langage. A l'inverse, l'étude des règles d'apparence conjointe des mots dans les énoncés (par exemple les textes littéraires) permet de faire des conjectures sur les normes associatives en usage au cours des époques précédentes. Ainsi on peut faire des conclusions sur la stabilité ou, au contraire, la labilité des normes associatives.

Quatrièmement, l'étude des réactions associatives a pour avantage d'offrir la possibilité d'envisager ses résultats comme le profil des images mentales spécifiques à la culture et la langues françaises du début du XXIe siècle, images mentales qui tiennent compte des connaissances intellectuelles et des impressions sensorielles reflétées par les mots. Il est extrèmement fructueux d'analyser et de comparer les réactions d'associations aux mêmes stimuli données par :

- les locuteurs natifs du français ;

- les personnes dont le français est la deuxième langue maternelle (dans une situation de bilinguisme précoce) ;

- les personnes étudiant le français en tant que langue étrangère.

Dans les deux derniers cas il est utile de comparer également avec leurs réactions au stimulus équivalent (sa traduction la plus proche) dans la langue maternelle (langue première) de ces personnes.

A titre d'exemple on peut citer les réactions données par des Russes, des Anglais et des Russes étudiant l'anglais au mot « eau» (водa 'voda' vs water). 


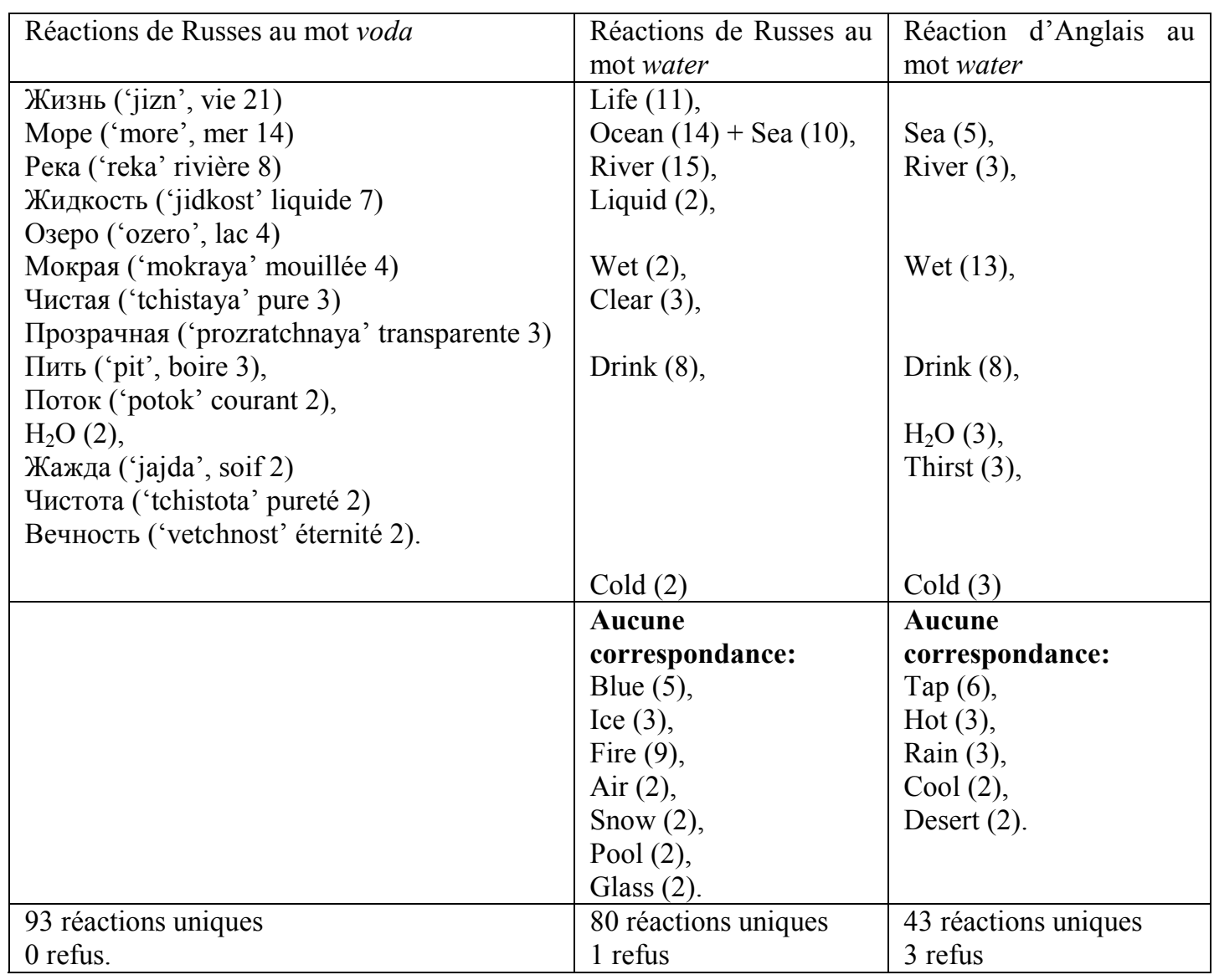

Tableau $\mathrm{N}^{\circ}$ 3. Comparaison des réactions associatives en langue maternelle et langue étrangères

\section{Conclusions}

L'analyse comparative du Dictionnaire Associatif du Français et du Dictionnaire Associatif du Russe permettra non seulement de déterminer la spécificité de ces deux consciences linguistiques mais de mettre en valeur ce qu'elles ont en commun et qui conditionne la réussite de la communication interculturelle et la traduction. Une comparaison ultérieure avec les consciences linguistiques anglaise, allemande, chinoise etc. basée sur les dictionnaires en cours de création rendra possible l'élaboration de recommandations tenant compte des spécificités ethniques nécessaires à une meilleure communication au niveau international.

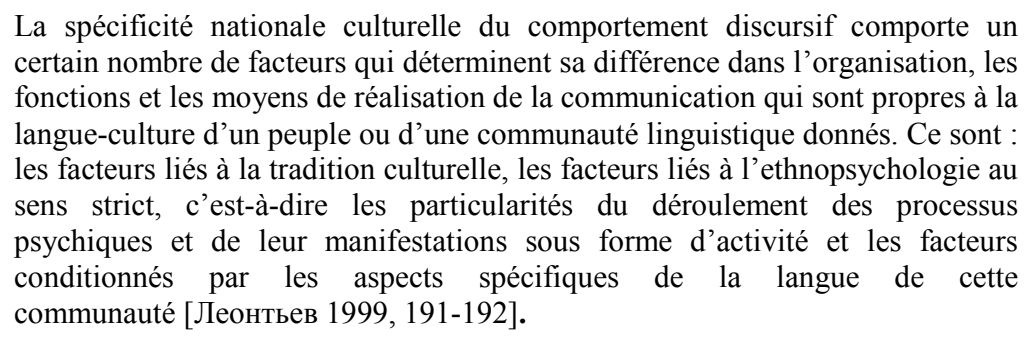

Les différentes disciplines complémentaires (ethnologie, psychologie, sociologie) dans le cadre desquelles se développe ce projet lui confèrent des perspectives variées. Dans le domaine de la sémantique et la lexicographie il est intéressant de comparer les résultats obtenus et ceux fixés dans 
les dictionnaires habituels, créés par des auteurs individuels et qui contiennent des définitions qui permettent au lecteur de recreer une connaissance de type théorique. Cette connaissance théorique est cependant un modèle non adéquat d'un locuteur profane parce qu'elle est le résultat d'une procédure d'analyse basée sur la déduction logique linéaire, alors que la connaissance profane doit se recréer au cours de processus non linéaires, parallèles, soumis à divers niveaux de contrôle conscient et inconscient. Les modèles en réseau sont les plus adéquats à l'heure actuelle, ils montrent que la recherche du mot demandé lors de la production et la compréhension du discours s'accompagnent de plusieurs liens avec d'autres mots. La compréhension d'un mot ou d'une expression se déroule en grande partie de manière inconsciente et la connaissance qui se forme diffère d'une connaissance théorique, reversible et déterministe.

Une expérience associative libre permet d'obtenir une information sur les équivalents psychologiques des «champs sémantiques» et de découvrir quels liens sémantiques existent effectivement dans l'esprit des locuteurs, ce qui rend cette expérience intéressante non seulement pour le psychologue ou le psycholinguiste mais aussi pour le linguiste qui s'intéresse à la sémantique. Ainsi si les dictionnaires traditionnels faits par des linguistes représentent en grande partie la description d'une conscience linguistique individuelle, les dictionnaires associatifs sont un moyen de décrire la conscience linguistique collective et usuelle des véritables locuteurs. Le dictionnaire associatif reflète d'une manière plus adéquate la conscience linguistique reelle et moyenne.

Par ailleurs, il est prévu d'étendre dans une deuxième étape du projet l'analyse de l'image du monde de locuteurs provenants d'autres régions de la Francophonie (Suisse, Belgique, Canada, Afrique) et de la comparer avec la conscience linguistique des Français de France. Les résultats obtenus formeront également la base d'une série de manuels de Français Langue Etrangère de nouvelle génération, de traduction du français et vers le français, de communication interculturelle.

\section{Références bibliographiques}

Kent G. H., Rosanoff A. J. (1910). A study of association in insanity. American Journal of Insanity, v. 67, No. $1-2$.

Kiss G., Armstrong C., Milroy R.(1972) The Associative Thesaurus of English. Edinburg : Univ. of Edinburg.

L. Postman (1970) Norms in word association. N.Y.: Academic Press.

Puig S., Karaulov Yu., Cherkasova G.(2001) Normas asociativas del espagnol y del ruso. Moscu - Madrid 2001

Russel W. A., Jenkins J. J. (1954) The complete Minnesota norms for responses to 100 words from the KentRosanoff word association test. Minneapolis: University of Minnesota.

Tarasov, E. F., Ufimtseva, N. V. (1999) Ethnopsycholinguistik. Kulturunterschiede. Frankfurt (Main).

Thumb A., Marbe K. (1901) Experimentelle Untersuchungen über die psychologischen Grundlagen der sprachlichen Analogiebildung. Leipzig.

Ufimtseva, N. V. (1999) Ethno-cultural specifity of images of language consciousness of contemporary Russian. 5-th International Congress of ISAPL, Proceedings, Faculdade de letras da Universidade do Porto, Porto, s. 757- 761.

Выготский Л .С. (1999) Мышление и речь (La Pensée et le discours). М.: Лабиринт.

Леонтьев А. А.(1965) Слово в речевой деятельности (Le mot et l'activité langagière).М.: Комкнига 2006

Леонтьев А.А. (1967) Психолингвистика (La psycholinguistique). M.

Леонтьев А. А. (1977) Словарь ассоциативных норм русского языка (Dictionnaire des normes associatives du russe). $M$.

Леонтьев А.А. (1997) Основы психолингвистики (Principes de Psycholinguistique). М.: Смысл, 1999. 
Тарасов Е. Ф. (2002) Диалог культур в зеркале языка (Le dialogue des cultures dans le miroir de la langue). Встречи этнических культур в зеркале языка. М.: Наука, с. 110-120.

Тарасов Е. Ф. (2004) Языковое сознание (La conscience linguistique). Вопросы психолингвистики, № 2, с. 34-47.

Тарасов Е.Ф. (1996) Межкультурное общение - новая онтология анализа языкового сознания (La communication interculturelle comme nouvelle ontologie de la conscience linguistique). Этнокультурная специифика языкознания. М., 1996.

Тарасов Е.Ф. (1998) К построению теории межкультурного общения (Vers la création d'une théorie de la communication interculturelle). Языковое сознание: формирование и функционирование М. 1998.

Уфимцева Н. В. (2000) Языковое сознание и образ мира славян (Conscience linguistique et image du monde des Slaves). Языковое сознание и образ мира. М.: Институт языкознания РАН, с. 207-219.

Уфимцева Н. В. (2002) Сознание, слово, культура (Conscience, mot, culture). Litora Psycholinguistica, Sofiia: SEMA RSh, c. 142-149.

Уфимцева Н. В. (2003) Языковое сознание как отображение этносоциокультурной реальности (La conscience linguistique comme reflet de la réalité ethnosocioculturelle). Вопросы психолингвистики. № 1, c. $102-110$.

Уфимцева Н. В. (2004) Ассоциативный тезаурус русского языка как модель языкового сознания русских (Le thésaurus associatif de la langue russe en tant que modèle de la conscience linguistique russe). Языковое сознание: теоретические и прикладные аспекты. М.: Институт языкознания РАН, с. 188202.

Уфимцева Н. В. и др.(2004) Ассоииативные нормы русского и немецкого языков (Les normes associatives du russe et de l'allemand). М.-Воронеж: Ияз РАН - ВГУ.

Уфимцева Н. В., Черкасова Г. А., Караулов Ю. А., Тарасов Е. Ф. (2004) Славянский ассоииативный словарь (Dictionnaire associatif slave). М.: МГЛУ-Ияз РАН. 